\title{
Meat consumption in the European Prospective Investigation into Cancer and Nutrition (EPIC) cohorts: results from 24-hour dietary recalls
}

\author{
J Linseisen ${ }^{1,2, *}$, E Kesse ${ }^{3}, \mathrm{~N}$ Slimani ${ }^{4}$, HB Bueno-de-Mesquita ${ }^{5}$, MC Ocké $^{5}, \mathrm{G} \mathrm{Skeie}^{6}$, \\ M Kumle ${ }^{6}, M$ Dorronsoro Iraeta ${ }^{7}, P$ Morote Gómez ${ }^{8}$, L Janzon $^{9}$, P Stattin ${ }^{10}$, AA Welch ${ }^{11}$, \\ EA Spencer ${ }^{12}, \mathrm{~K}$ Overvad $^{13}$, A Tiønneland ${ }^{14}$, F Clavel-Chapelon $^{3}$, AB Miller $^{2}, \mathrm{~K}_{\text {Klipstein- }}$

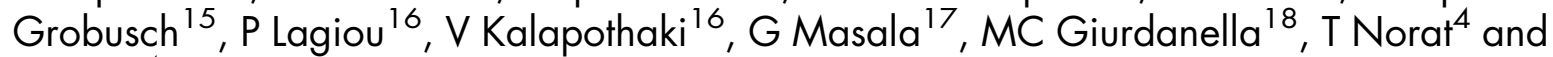 \\ E Riboli ${ }^{4}$ \\ 'Unit of Human Nutrition and Cancer Prevention, Technical University of Munich, Alte Akademie 16, D-85405 \\ Freising-Weihenstephan, Germany: ${ }^{2}$ Division of Clinical Epidemiology, German Cancer Research Centre, \\ Heidelberg, Germany: ${ }^{3}$ INSERM, E3N-EPIC Group, Institute Gustave Roussy, Villejuif, France: ${ }^{4}$ Unit of Nutrition, \\ International Agency for Research on Cancer, Lyon, France: ${ }^{5}$ Department of Chronic Diseases Epidemiology, \\ National Institute for Public Health and the Environment, Bilthoven, The Netherlands: Institute of Community \\ Medicine, University of Tromsø, Norway: ${ }^{7}$ Public Health Division of Gipuzkoa, Department of Health of the Basque \\ Government, San Sebastian, Spain: ${ }^{8}$ Public Health Directorate, Health Council and Health Services Asturias, \\ Oviedo, Spain: ${ }^{9}$ Department of Community Medicine, Lund University, Malmö, Sweden: ${ }^{10}$ Department of Urology \\ and Andrology, Umeå University Hospital, Sweden: ${ }^{11}$ Institute of Public Health, School of Clinical Medicine, \\ University of Cambridge, UK: ${ }^{12}$ Cancer Research UK, Epidemiology Unit, University of Oxford, UK: ${ }^{13}$ Department of \\ Epidemiology and Social Medicine, University of Aarhus, Denmark: ${ }^{14}$ Institute of Cancer Epidemiology, Danish \\ Cancer Society, Copenhagen, Denmark: ${ }^{15}$ Department of Epidemiology, German Institute of Human Nutrition, \\ Potsdam-Rehbrücke, Germany: ${ }^{16}$ Department of Hygiene and Epidemiology, School of Medicine, University of \\ Athens, Greece: ${ }^{17}$ Molecular and Nutritional Epidemiology Unit, CSPO, Scientific Institute of Tuscany, Florence, Italy: \\ ${ }^{18}$ Cancer Registry, 'Civile - M.P. Arezzo' Hospital, Ragusa, Italy
}

\begin{abstract}
Objective: To evaluate meat intake patterns in the European Prospective Investigation into Cancer and Nutrition (EPIC) cohorts.

Design and setting: 24-Hour dietary recalls were assessed within the framework of a prospective cohort study in 27 centres across 10 European countries by means of standardised computer-assisted interviews.

Subjects: In total, 22924 women and 13031 men aged 35-74 years.

Results: Mean total meat intake was lowest in the 'health-conscious' cohort in the UK ( 15 and $21 \mathrm{~g} \mathrm{day}^{-1}$ in women and men, respectively) and highest in the north of Spain, especially in San Sebastian (124 and $234 \mathrm{~g} \mathrm{day}^{-1}$, respectively). In the southern Spanish centres and in Naples (Italy), meat consumption was distinctly lower than in the north of these countries. Central and northern European centres/countries showed rather similar meat consumption patterns, except for the British and French cohorts. Differences in the intake of meat sub-groups (e.g. red meat, processed meat) across EPIC were even higher than found for total meat intake. With a few exceptions, the Mediterranean EPIC centres revealed a higher proportion of beef/veal and poultry and less pork or processed meat than observed in central or northern European centres. The highest sausage consumption was observed for the German EPIC participants, followed by the Norwegians, Swedish, Danish and Dutch.

Conclusions: The results demonstrate distinct differences in meat consumption patterns between EPIC centres across Europe. This is an important prerequisite for obtaining further insight into the relationship between meat intake and the development of chronic diseases.
\end{abstract}

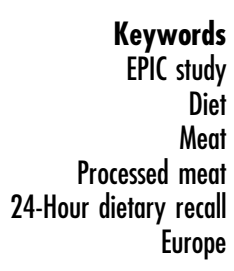

Keywords C study

Diet

Meat Europe
More than for other basic food groups in human nutrition, meat consumption patterns vary considerably over time, cultures and the personal situation ${ }^{1,2}$. Besides complete or selective avoidance of meat intake for ethical or religious reasons, socio-economic factors and health aspects are likely to be the most prominent factors in determining meat consumption patterns. In terms of health effects, both benefits and risks associated with meat intake have 
been identified ${ }^{1}$. Meat is an excellent source of highquality protein, haem iron and zinc, and may have a large impact on meeting the requirement of further nutrients, e.g. vitamins of the $\mathrm{B}$ group (particularly $\left.\mathrm{B}_{12}\right)^{1}$. On the other hand, the original constituents of meat (e.g. saturated fatty acids, cholesterol and iron) as well as added compounds or compounds produced during the preservation or preparation of meat (e.g. salt, nitrate/ nitrite, smoke, heterocyclic amines) may be harmful for human health ${ }^{3}$. For the two major chronic diseases prevalent in the Western world, coronary heart disease (CHD) and cancers of different sites, the role of meat and meat constituents has long been a matter of debate. In $\mathrm{CHD}$, it is mainly the high proportion of fat calories and saturated fatty acids provided by meat that may affect plasma cholesterol concentrations as well as other risk factors of CHD (obesity, hypertension) ${ }^{4}$. Hypotheses concerning the role of meat in cancer development refer predominantly to the formation of potentially carcinogenic compounds during food preparation and cooking such as heterocyclic amines, polycyclic aromatic hydrocarbons and $\mathrm{N}$-nitroso compounds ${ }^{3,5}$. There is evidence for an increased risk of cancers of the colon, rectum, breast, prostate, pancreas and kidney with increasing meat intake $e^{6,7}$. At least for colorectal cancer and possibly also for breast cancer, the association seems to be more consistent for red meat and processed meat (mainly sausages, ham and bacon) than for overall meat intake ${ }^{5,8-10}$. As a further causal agent, iron can act as a prooxidant and its linkage to the development of colorectal cancer as well as CHD has been suggested ${ }^{11-13}$. Additionally, in inflammatory states where the amount and the ratio of different $n-6$ and $n-3$ fatty acids as precursors of prostanoids may be important (e.g. rheumatoid arthritis), there is discussion on the optimal amount of meat (fat) intake due to its high content of arachidonic acid (C20:4n-6) $)^{14}$.

In epidemiological studies on meat intake and disease risk, the term 'meat' is not always well or equally defined $^{7}$. Some studies include poultry and fish in the definition of meat while others differentiate between red meat, white meat, processed meat and fish. In general, red meat refers to beef, lamb and pork; processed meat refers to sausages, hamburgers, smoked, cured and salted meat and canned meat ${ }^{7}$. For veal, the situation is unclear and the category may depend upon the age at slaughter and feeding practices. Cross-sectional differences in meat intake and changes over time are usually described by means of food balance sheets and household survey data across Europe ${ }^{15,16}$. The assessment of differences in meat consumption across European countries by means of a standardised dietary intake method has been reported for elderly women only $^{17}$. The aim of this paper was to compile data on dietary meat intake across European countries and centres participating in the European Prospective
Investigation into Cancer and Nutrition (EPIC). In this paper, unique definitions and assessment methods were considered and optimised to obtain reliable and comparable estimates of dietary meat intake.

\section{Subjects}

The EPIC cohort study includes about half a million subjects from 10 European countries (France, Italy, Spain, Greece, the Netherlands, the UK, Germany, Denmark, Sweden and Norway) $)^{18}$. Information on the usual diet of all participants has been assessed by country-specific instruments, largely food-frequency questionnaires. In order to adjust (at the group level) for systematic measurement error between countries, highly standardised 24-hour recalls were performed in a sub-sample of the cohort as an additional dietary measurement ${ }^{18,19}$. The present estimate is based on these 24-hour dietary recalls from 22924 women and 13031 men participating in the EPIC calibration study between 1995 and 1998 (except Norway: 1999-2000). The distribution of study participants over the 27 study centres in 10 European countries is given in Tables 1-4; in France, Norway, Utrecht (The Netherlands) and Naples (Italy) women only were recruited. In the UK, a special group of 'health-conscious' people was included in the EPIC study. This group includes vegans as well as ovo-lacto vegetarians, fish eaters (consuming fish but no meat) and meat eaters. All participants included in the present evaluation were in the age range of 35-74 years at recruitment. A detailed description of further characteristics of the study participants is given elsewhere in this supplement ${ }^{19}$. Unless otherwise specified, the terms 'middle European' and 'northern European' refer to EPIC centres in France, Germany, The Netherlands and the UK and to EPIC centres in Denmark, Sweden and Norway, respectively. 'Mediterranean' EPIC centres are those from Greece, southern Spain and southern Italy.

\section{Methods}

A computerised 24-hour dietary recall interview program, EPIC-SOFT, was developed as a calibration instrument by the International Agency for Research on Cancer in collaboration with all EPIC study centres ${ }^{20,21}$. The program was adapted for each participating country in terms of foods and recipes included. EPIC-SOFT provides a common structure and interview interface for an optimised standardisation of the dietary interview procedure within and between EPIC centres. On the basis of a predefined list of food groups and food sub-groups, the countries filled in the single food items expected to be consumed by their participants. The open design allowed iterative modification of the food item list. Furthermore, national data on the energy, fat, carbohydrate and alcohol contents of the food items were inserted to allow a rapid 







\begin{tabular}{|c|c|c|c|c|c|c|c|c|c|c|c|c|c|}
\hline$\Phi$ & & 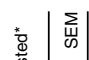 & $\hat{o}$ & 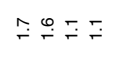 & 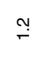 & 욤 & $\stackrel{\infty}{0}$ & $\stackrel{\infty}{\circ}$ & $\stackrel{\dddot{N}}{\longrightarrow}$ & & $\hat{o}$ & $\hat{o} \hat{o}$ & $\bar{\sigma}$ \\
\hline $\bar{z}$ & 离 & 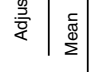 & مִ & 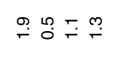 & $\stackrel{\circ}{i}$ & 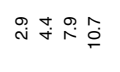 & $\stackrel{\Gamma}{i} \stackrel{m}{+}$ & ò & $\stackrel{\llcorner}{+}$ & & $\underset{\omega}{N} \overline{0}$ & & \\
\hline & & क्षे & $\stackrel{\sim}{=}$ & 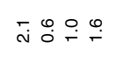 & 广̀ & 品 & 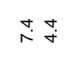 & $\stackrel{\circ}{-}$ & $\stackrel{\leftrightarrow}{+}$ & & 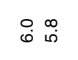 & & \\
\hline & & 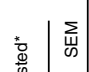 & $\stackrel{\square}{\leftarrow}$ & एँ & $\stackrel{\text { N }}{\sim}$ & 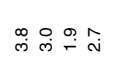 & $\stackrel{\leftrightarrow}{\stackrel{+}{+}}$ & $\stackrel{\circ}{\leftarrow}$ & $\stackrel{n}{N}$ & & $m=\overline{\mathrm{N}}$ & & \\
\hline$\varrho$ & 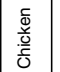 & 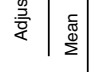 & $\stackrel{\mathscr{N}}{\stackrel{N}{ }}$ & 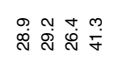 & बूं & 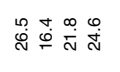 & $\stackrel{\infty}{\infty} \stackrel{\infty}{\infty}$ & $\stackrel{\hat{\omega}}{\stackrel{\omega}{ }}$ & $\stackrel{\text { ì }}{ }$ & $\infty$ & 吊号 & & \\
\hline & & 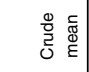 & 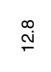 & 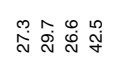 & $\stackrel{\circ}{\sim}$ & 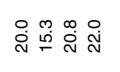 & $\stackrel{0}{\infty} \dot{\infty}$ & 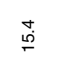 & $\stackrel{\infty}{\stackrel{N}{N}}$ & 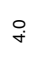 & 余吊 & $\stackrel{N}{\stackrel{N}{\pi}}$ & \\
\hline & \pm & 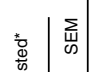 & $\stackrel{\circ}{\leftarrow}$ & ळ. & $\stackrel{\infty}{N}$ & 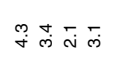 & $\stackrel{\circ}{=}$ & $\stackrel{\infty}{\leftarrow}$ & $\stackrel{\infty}{\sim}$ & & 님 & & \\
\hline & 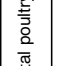 & 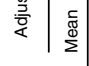 & $\stackrel{\infty}{+}$ & 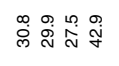 & 今. & 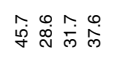 & $\stackrel{\infty}{\infty} \stackrel{0}{\infty}$ & $\underset{\oplus}{\stackrel{\oplus}{\circ}}$ & 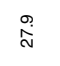 & & 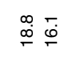 & 界 & \\
\hline & 흥 & 옳 & $\stackrel{\infty}{ \pm}$ & 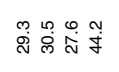 & 户ें & 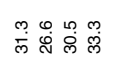 & 悉怘 & فㅇ & $\stackrel{\sim}{\infty}$ & $\stackrel{\circ}{\dot{q}}$ & 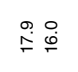 & 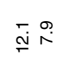 & \\
\hline & & 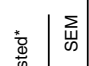 & $\stackrel{\circ}{\circ}$ & 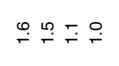 & 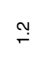 & 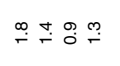 & $\hat{o} \hat{o}$ & $\hat{o}$ & $\check{F}$ & กี & : & $\stackrel{0}{\circ}$ & \\
\hline & 殄 & 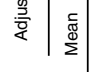 & is & 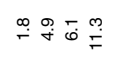 & $\bar{q}$ & 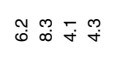 & $\stackrel{0}{\stackrel{\circ}{\circ}}$ & $\stackrel{m}{\sim}$ & $\stackrel{5}{亡}$ & $\stackrel{\circ}{\circ}$ & 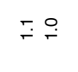 & & \\
\hline & & 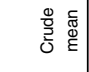 & $\stackrel{\circ}{\circ}$ & $\hat{N} \stackrel{+}{*} \stackrel{\circ}{\circ} \stackrel{0}{0}$ & $\stackrel{\infty}{+}$ & ma & $\stackrel{\oplus}{\dot{P}} \stackrel{m}{=}$ & $\stackrel{\circ}{\dot{-}}$ & $\stackrel{+}{\leftarrow}$ & $\stackrel{8}{\circ}$ & 冚 & 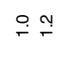 & \\
\hline & & 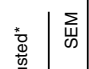 & $\hat{o}$ & : & $\stackrel{m}{+}$ & 언 $\stackrel{0}{i} \stackrel{\circ}{\leftarrow}$ & 央 & $\stackrel{\infty}{\infty}$ & $\stackrel{m}{=}$ & ఫ & $\bar{z}$ & $\hat{o} \hat{o}$ & \\
\hline & 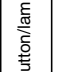 & 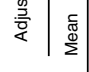 & $\hat{\imath}$ & 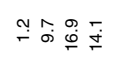 & $\stackrel{\circ}{+}$ & $\hat{m}$ ำ & $\cong \stackrel{m}{0}$ & $\overline{\mathrm{i}}$ & $\stackrel{t}{\omega}$ & $\mp$ & $\hat{\text { No }}$ & & \\
\hline & $\frac{\bar{\Sigma}}{\Sigma}$ & 홀 ₹ & $\stackrel{\infty}{\infty}$ & 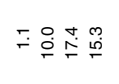 & $\stackrel{9}{\dot{r}}$ & 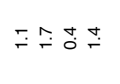 & $\stackrel{n}{\circ}$ & $\stackrel{\text { ì }}{ }$ & $\stackrel{\mathscr{0}}{0}$ & $\stackrel{\infty}{\circ}$ & $\stackrel{\circ \sim N}{\sim}$ & $\stackrel{m}{i} \stackrel{\circ}{-}$ & \\
\hline & & 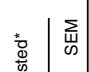 & $\stackrel{m}{+}$ & $\bar{m} \bar{N} \bar{N} \bar{N} \bar{N}$ & $\stackrel{\infty}{i}$ & 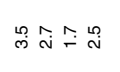 & $\stackrel{+\infty}{\stackrel{m}{\sim}}$ & $\stackrel{\circ}{\sim}$ & 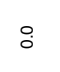 & $\stackrel{\circ}{\circ}$ & 꼼 & $\stackrel{\infty}{\stackrel{\infty}{*}}$ & \\
\hline & $\stackrel{\bar{\Phi}}{\stackrel{5}{\supset}}$ & 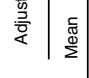 & $\stackrel{\infty}{\stackrel{\infty}{N}}$ & 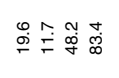 & $\infty_{\infty}^{\infty}$ & 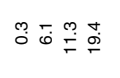 & $\stackrel{\square}{\circ}$ & $\stackrel{+}{\check{5}}$ & $\stackrel{\circ}{\circ}$ & $\therefore$ & 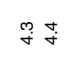 & & \\
\hline & & 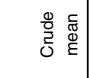 & ث̊. & 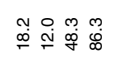 & 今. & 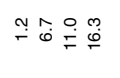 & $\stackrel{\text { i }}{0}$ & $\stackrel{\infty}{\sim}$ & $\therefore$ & $\stackrel{0}{0}$ & $\bar{f} \overline{\dot{\sigma}}$ & $\stackrel{\circ}{\dot{\gamma}}$ & \\
\hline & & 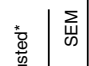 & $\stackrel{+}{\dot{t}}$ & 용 ल용 & $\stackrel{\leftrightarrow}{\mathrm{i}}$ & 闭 & & $\stackrel{\circ}{\Gamma}$ & $\stackrel{\mathscr{N}}{\mathrm{i}}$ & f & $\stackrel{+}{\stackrel{i}{\sim}}$ & $\stackrel{+}{\stackrel{4}{\rightleftarrows}}$ & \\
\hline & $\begin{array}{c}\bar{\Phi} \\
\Phi\end{array}$ & 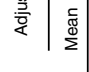 & $\stackrel{\text { ஸे }}{ }$ & : & $\stackrel{\infty}{\circ}$ & 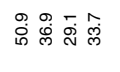 & $\stackrel{\infty}{\stackrel{\infty}{\rho} \dot{\infty}}$ & $\stackrel{\circ}{\sim}$ & ㅇ. & $\stackrel{\infty}{\infty}$ & 总芦 & 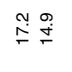 & పั \\
\hline & & 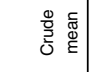 & $\stackrel{\circ}{\check{1}}$ & $\stackrel{\circ}{\circ}=\stackrel{\circ}{\circ} \stackrel{\circ}{=}$ & $\stackrel{\leftrightarrow}{\circ}$ & 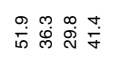 & 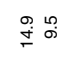 & $\stackrel{\stackrel{d}{N}}{ }$ & $\stackrel{\infty}{\infty}$ & $\stackrel{\infty}{\infty}$ & 邑 & 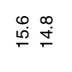 & \\
\hline$\overline{\bar{c}}$ & & 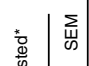 & $\stackrel{\circ}{\leftarrow}$ & 웅 & $\stackrel{9}{\mathrm{i}}$ & 号 & $\stackrel{\infty}{-\stackrel{0}{+}}$ & $\stackrel{9}{\longrightarrow}$ & $\stackrel{\leftrightarrow}{\mathrm{i}}$ & $\stackrel{+}{\omega}$ & 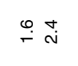 & $\stackrel{0}{\leftarrow} \stackrel{0}{-}$ & \\
\hline$\frac{\varepsilon}{\bar{\sigma}}$ & 홍 & 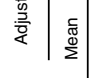 & 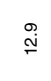 & 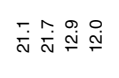 & $\stackrel{\infty}{\infty}$ & 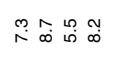 & 占号灾 & $\bar{i}$ & 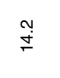 & $\stackrel{q}{\mathcal{g}}$ & 承 $\stackrel{0}{\mathrm{~N}}$ & 尺्र & \\
\hline 2 & & & $\stackrel{\mathscr{\rho}}{\stackrel{\rho}{\rho}}$ & 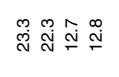 & $\stackrel{\infty}{\infty}$ & 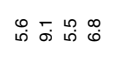 & 第第 & $\stackrel{\circ}{\infty}$ & $\stackrel{\circ}{\underline{\rho}}$ & $\stackrel{\mathscr{\rho}}{\infty}$ & 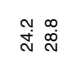 & 임 & \\
\hline$\frac{\pi}{0}$ & & 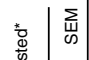 & $\stackrel{\leftrightarrow}{i}$ & 용 & 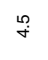 & 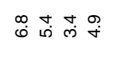 & & $\stackrel{\infty}{i}$ & $\stackrel{+}{\dot{q}}$ & $\underset{\infty}{\infty}$ & Ni & N & \\
\hline 劳 क & 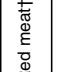 & & 离 & 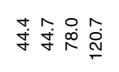 & $\bar{\infty}$ & 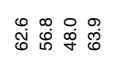 & 品 & $\begin{array}{l}\infty \\
\stackrel{\infty}{6}\end{array}$ & 염 & r & 商 & 乫 & \\
\hline$\frac{\pi}{\sigma} \frac{\pi}{\pi}$ & & & $\stackrel{\infty}{+j}$ & 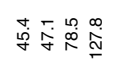 & 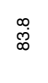 & 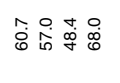 & 怘虽 & 离 & 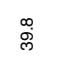 & $r$ & 番 & 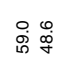 & \\
\hline$\overline{0}$ & 志 & & $\stackrel{\infty}{i}$ & 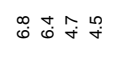 & 우 & 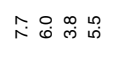 & $\bar{\infty} \stackrel{\infty}{\dot{N}}$ & 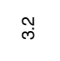 & $\stackrel{9}{+}$ & 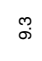 & $\hat{\mathrm{i}} \stackrel{\mathfrak{q}}{\mathrm{N}}$ & $\stackrel{\infty}{i} \hat{i}$ & $\bar{\sigma}$ \\
\hline 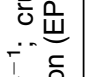 & 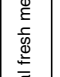 & 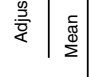 & $\hat{\circ}$ & 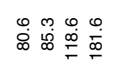 & 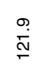 & 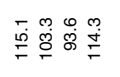 & 天芒宽 & $\bar{\infty}$ & 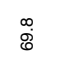 & $\stackrel{\rho}{\longrightarrow}$ & 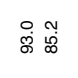 & & \\
\hline s. & 咟 & 을 윯 & $\hat{8}$ & 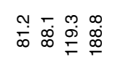 & 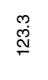 & 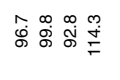 & 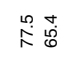 & $\underset{\infty}{\grave{\infty}}$ & $\stackrel{0}{\circ}$ & & $\begin{array}{c}\substack{\infty \\
\infty \\
\infty} \\
\infty\end{array}$ & & \\
\hline 음 & & \begin{tabular}{l|l}
$\frac{5}{4}$ \\
$\frac{\mathrm{d}}{\mathrm{w}}$ \\
$\frac{\mathrm{w}}{\mathrm{w}}$
\end{tabular} & $\bar{m}$ & 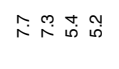 & $\underset{\omega}{\infty}$ & 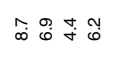 & & $\stackrel{\circ}{\circ}$ & $\stackrel{0}{\infty}$ & & $\bar{m} \stackrel{\infty}{\dot{q}}$ & & \\
\hline 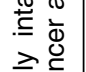 & 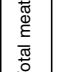 & & $\underset{\substack{\infty \\
\infty}}{\infty}$ & 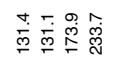 & 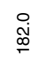 & 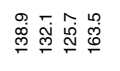 & 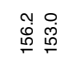 & 兽 & $\stackrel{-\infty}{\stackrel{\infty}{\circ}}$ & & 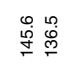 & 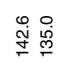 & \\
\hline c & & & $\underset{\substack{\infty \\
\infty}}{\infty}$ & 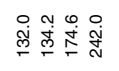 & $\underset{\Phi}{\tilde{D}}$ & 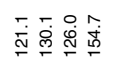 & 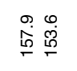 & $\stackrel{\hat{\theta}}{\circ}$ & 号 & & 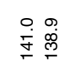 & & \\
\hline 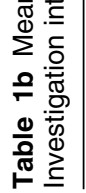 & & 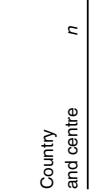 & $\stackrel{N}{\underline{m}}$ & 志喿表察 & $\stackrel{\infty}{\infty}$ & 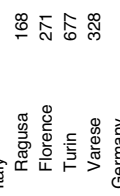 & 浔 & ণ্ণ & 草 & $=$ & 总 & 可尌 & $\begin{array}{l}\frac{\pi}{6} \\
1 \\
\frac{1}{5}\end{array}$ \\
\hline
\end{tabular}




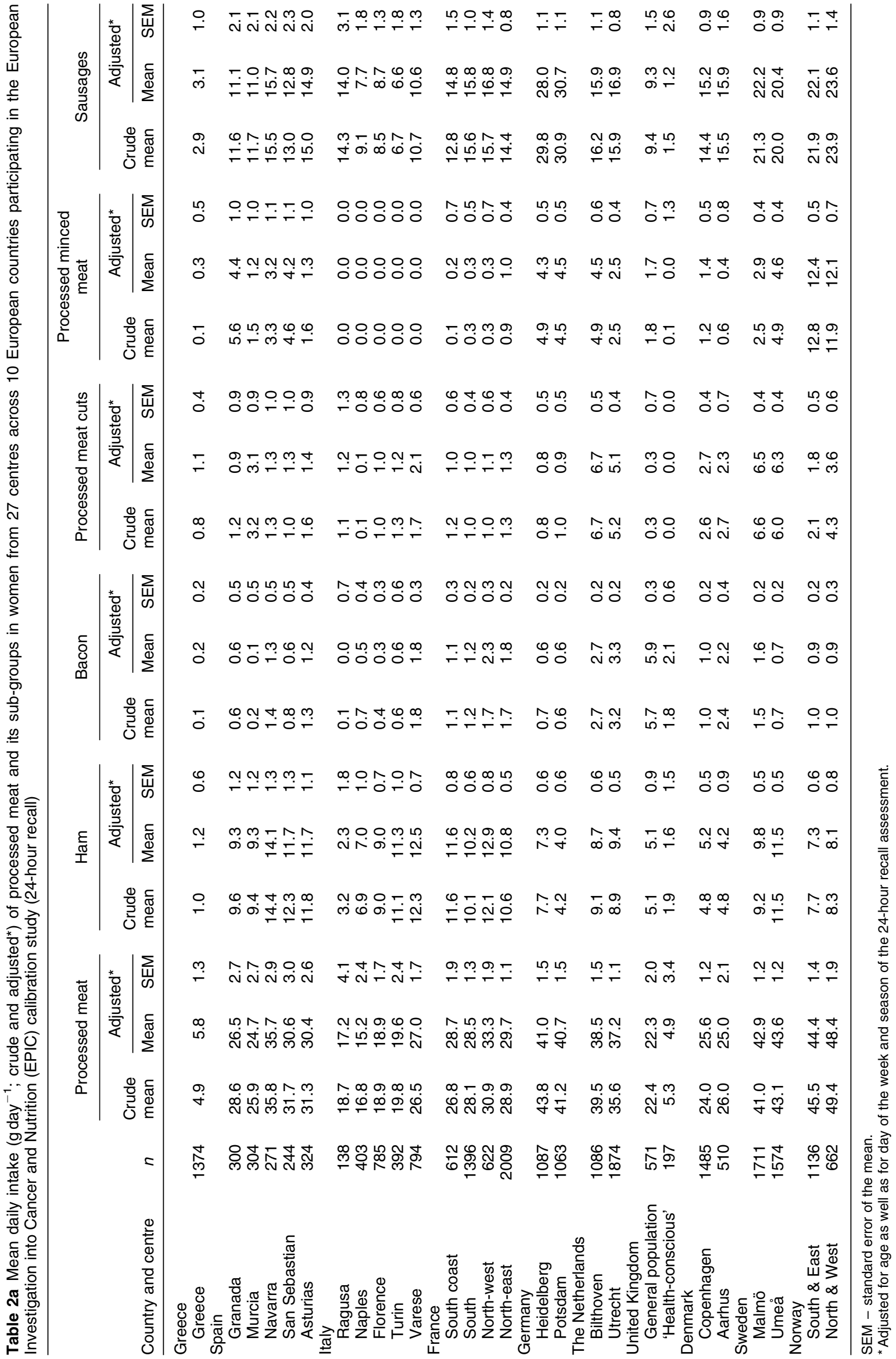




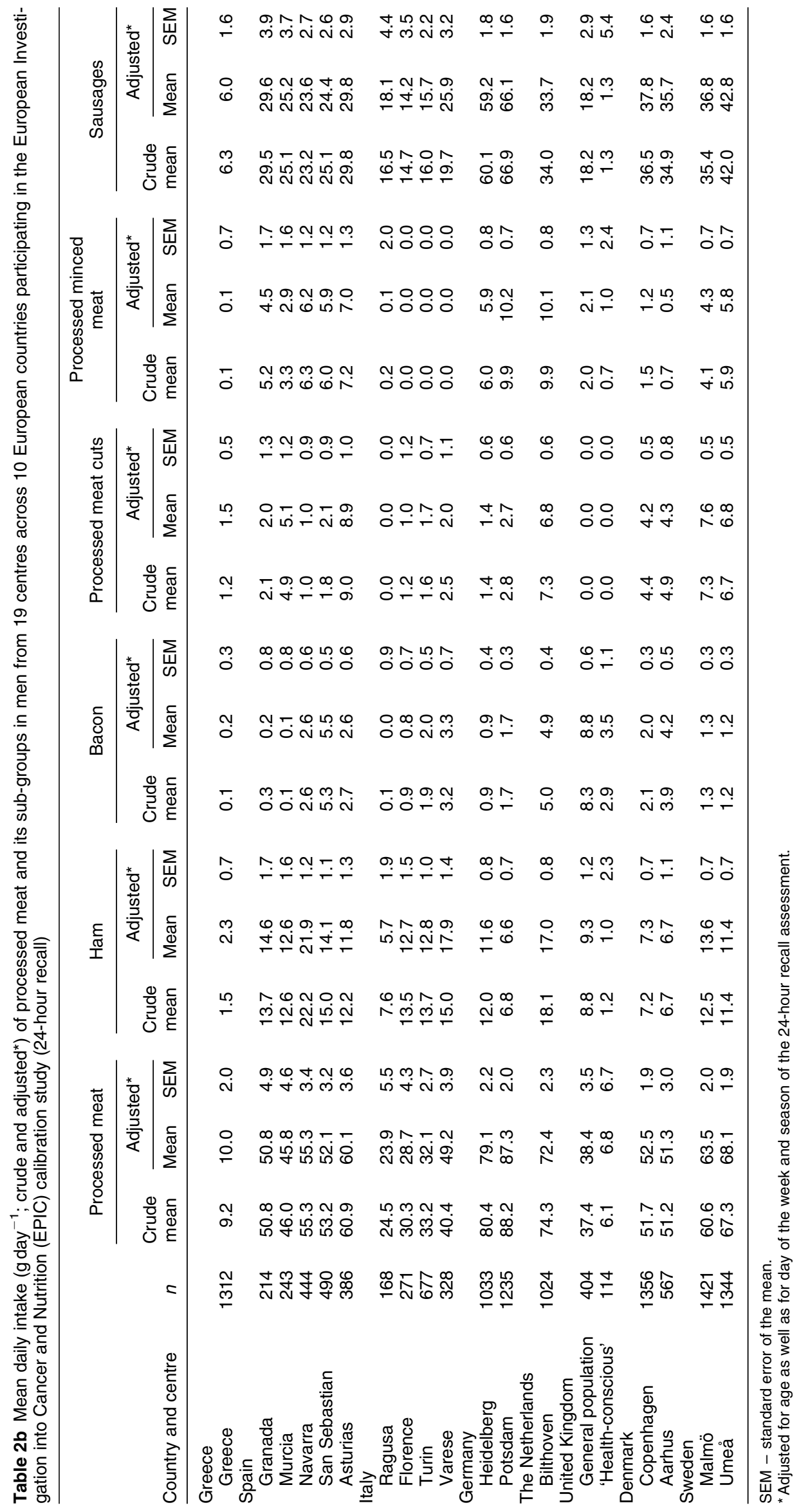


Meat consumption in EPIC

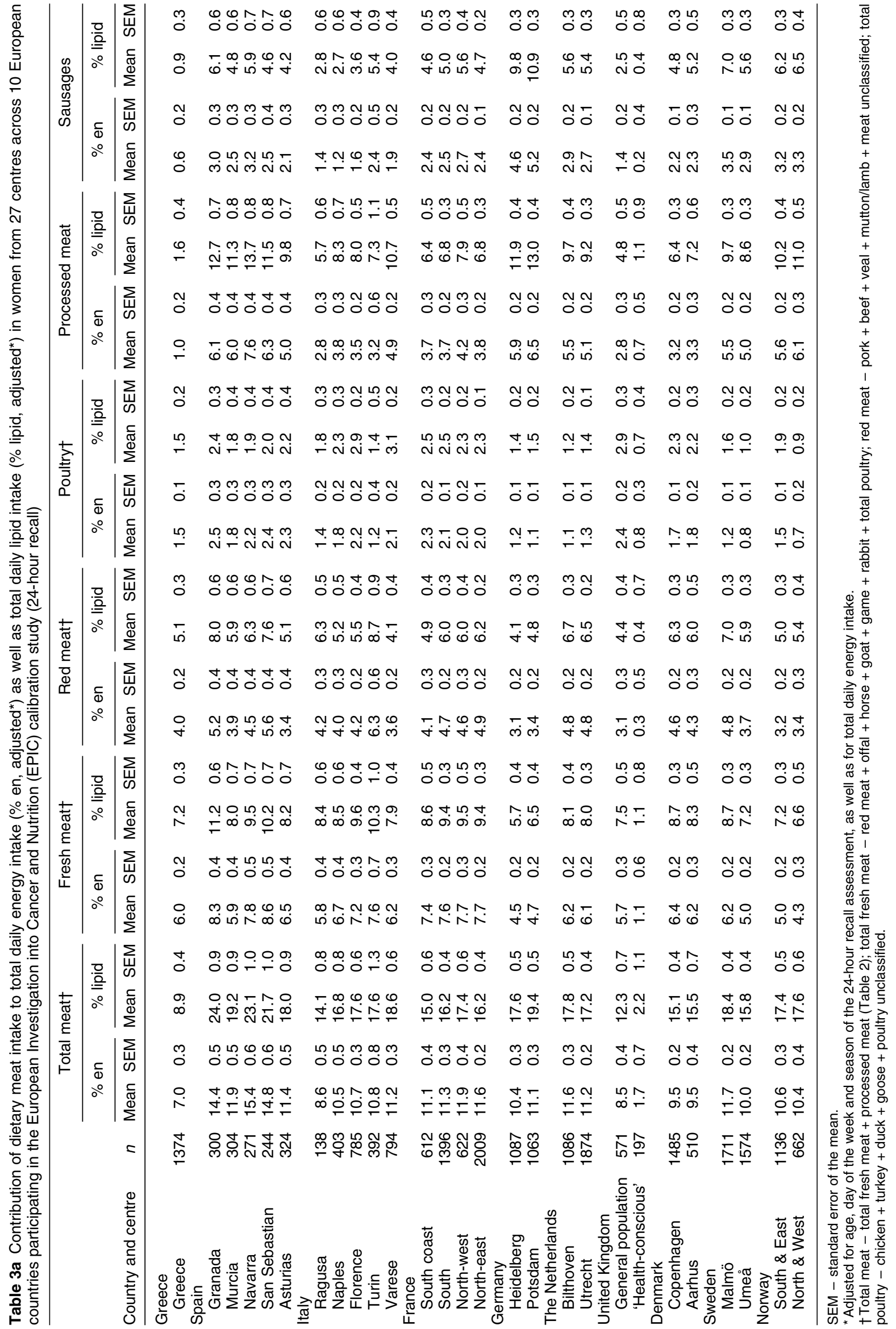


Table 4 Mean meat intake $\left(\mathrm{g} \mathrm{day}^{-1}\right.$ ) by recall day, age, body mass index (BMI), smoking, educational level and sports activity, adjusted* with and without inclusion of total energy intake, in women and men participating in the European Investigation into Cancer and Nutrition (EPIC) calibration study (24-hour recall)

\begin{tabular}{|c|c|c|c|c|c|c|c|c|c|c|}
\hline \multirow[b]{4}{*}{ Factor } & \multirow[b]{4}{*}{ Women $(n)$} & \multirow[b]{4}{*}{$\operatorname{Men}(n)$} & \multicolumn{8}{|c|}{ Total meat intake $\left(\mathrm{g} \mathrm{day}^{-1}\right)$} \\
\hline & & & \multicolumn{4}{|c|}{ Without energy adjustment } & \multicolumn{4}{|c|}{ With energy adjustment } \\
\hline & & & \multicolumn{2}{|c|}{ Women } & \multicolumn{2}{|c|}{ Men } & \multicolumn{2}{|c|}{ Women } & \multicolumn{2}{|c|}{ Men } \\
\hline & & & Mean† & SEM & Mean† & SEM & Mean† & SEM & Mean† & SEM \\
\hline \multicolumn{11}{|l|}{ Recall day } \\
\hline Monday & 3960 & 2141 & $86.2^{\mathrm{a}}$ & 2.4 & $137.5^{\mathrm{a}}$ & 4.9 & $89.7^{\mathrm{a}}$ & 2.3 & $137.8^{\mathrm{a}}$ & 4.5 \\
\hline Tuesday & 4007 & 2212 & $85.3^{\mathrm{a}}$ & 2.4 & $128.5^{\mathrm{b}}$ & 4.8 & $88.5^{\mathrm{a}, \mathrm{c}}$ & 2.3 & $127.8^{\mathrm{b}}$ & 4.5 \\
\hline Wednesday & 3724 & 2064 & $81.4^{b, c}$ & 2.4 & $132.2^{\mathrm{b}}$ & 4.9 & $83.6^{\mathrm{b}}$ & 2.3 & $131.1^{\mathrm{b}}$ & 4.6 \\
\hline Thursday & 3205 & 1937 & $82.1^{\mathrm{a}, \mathrm{b}}$ & 2.5 & $126.9^{b}$ & 5.0 & $84.7^{b, c}$ & 2.4 & $125.8^{\mathrm{b}}$ & 4.6 \\
\hline Friday & 2178 & 1456 & $73.9^{c}$ & 2.7 & $130.7^{\mathrm{a}, \mathrm{b}}$ & 5.4 & $74.4^{\mathrm{d}}$ & 2.6 & $122.7^{b}$ & 4.9 \\
\hline Saturday & 2756 & 1525 & $93.0^{d}$ & 2.6 & $158.1^{c}$ & 5.3 & $88.0^{\mathrm{a}}$ & 2.5 & $138.5^{\mathrm{a}}$ & 4.9 \\
\hline Sunday & 3094 & 1696 & $104.4^{\mathrm{e}}$ & 2.5 & $157.3^{\mathrm{c}}$ & 5.1 & $100.6^{\mathrm{e}}$ & 2.4 & $148.5^{\mathrm{c}}$ & 4.7 \\
\hline \multicolumn{11}{|l|}{ Age (years) } \\
\hline $35-<45$ & 2231 & 1106 & $97.1^{a}$ & 2.8 & $154.0^{\mathrm{a}}$ & 5.6 & $94.8^{\mathrm{a}}$ & 2.7 & $139.0^{\mathrm{a}}$ & 5.2 \\
\hline $45-<55$ & 8597 & 3953 & $88.3^{\mathrm{b}}$ & 2.1 & $145.7^{b}$ & 4.5 & $88.2^{\mathrm{b}}$ & 2.0 & $137.7^{\mathrm{a}}$ & 4.1 \\
\hline $55-<65$ & 9003 & 5910 & $83.6^{\mathrm{C}}$ & 2.1 & $133.6^{c}$ & 4.4 & $84.8^{\mathrm{C}}$ & 2.0 & $130.6^{\mathrm{b}}$ & 4.0 \\
\hline $65-74$ & 3092 & 2062 & $77.5^{\mathrm{d}}$ & 2.5 & $121.7^{\mathrm{d}}$ & 5.4 & $80.5^{\mathrm{d}}$ & 2.4 & $125.3^{\mathrm{b}}$ & 4.9 \\
\hline \multicolumn{11}{|l|}{$\mathrm{BMI}\left(\mathrm{kg} \mathrm{m}^{-2}\right)$} \\
\hline$<20$ & 1385 & 139 & $75.9^{\mathrm{a}}$ & 3.0 & $126.7^{\mathrm{a}, \mathrm{b}}$ & 11.0 & $72.6^{\mathrm{a}}$ & 2.9 & $117.1^{\mathrm{a}}$ & 10.1 \\
\hline $20-25$ & 10879 & 3766 & $82.8^{\mathrm{b}}$ & 2.0 & $132.5^{\mathrm{b}}$ & 3.8 & $82.2^{\mathrm{b}}$ & 1.9 & $125.4^{\mathrm{a}}$ & 3.5 \\
\hline $25-30$ & 7363 & 6882 & $91.3^{\mathrm{C}}$ & 2.1 & $141.5^{\mathrm{a}}$ & 3.5 & $93.3^{c}$ & 2.0 & $137.7^{\mathrm{b}}$ & 3.2 \\
\hline$>30$ & 3296 & 2244 & $96.5^{\mathrm{d}}$ & 2.5 & $154.3^{\mathrm{c}}$ & 4.3 & $100.2^{d}$ & 2.4 & $152.4^{c}$ & 3.9 \\
\hline \multicolumn{11}{|l|}{ Smoking } \\
\hline Never & 13136 & 4196 & $84.2^{\mathrm{a}}$ & 1.9 & $135.9^{\mathrm{a}}$ & 4.6 & $83.4^{\mathrm{a}}$ & 1.8 & $130.0^{\mathrm{a}}$ & 4.2 \\
\hline In the past & 5189 & 5061 & $83.8^{\mathrm{a}}$ & 2.1 & $135.2^{\mathrm{a}}$ & 4.5 & $84.2^{\mathrm{a}}$ & 2.0 & $130.6^{\mathrm{a}}$ & 4.1 \\
\hline Currently & 4135 & 3558 & $96.2^{\mathrm{b}}$ & 2.2 & $145.2^{\mathrm{b}}$ & 4.6 & $96.7^{\mathrm{b}}$ & 2.1 & $138.9^{\mathrm{b}}$ & 4.2 \\
\hline \multicolumn{11}{|l|}{ Education } \\
\hline None & 964 & 749 & $80.6^{\mathrm{a}}$ & 3.6 & $148.0^{\mathrm{a}}$ & 6.1 & $82.6^{\mathrm{a}}$ & 3.4 & $142.7^{\mathrm{a}}$ & 5.6 \\
\hline Primary school & 6221 & 4344 & $92.6^{\mathrm{b}}$ & 1.5 & $146.4^{\mathrm{a}}$ & 3.7 & $93.8^{\mathrm{b}}$ & 1.5 & $141.4^{\mathrm{a}}$ & 3.4 \\
\hline Technical school & 4276 & 2913 & $92.2^{b, c}$ & 1.8 & $139.7^{b}$ & 3.8 & $92.8^{\mathrm{a}, \mathrm{b}}$ & 1.8 & $133.7^{b}$ & 3.5 \\
\hline Secondary school & 6297 & 2092 & $88.1^{\mathrm{c}}$ & 1.5 & $133.8^{\mathrm{b}}$ & 4.1 & $87.1^{\mathrm{a}, \mathrm{c}}$ & 1.5 & $131.9^{b}$ & 3.7 \\
\hline University degree & 4782 & 2804 & $82.7^{\mathrm{a}}$ & 1.7 & $121.1^{\mathrm{c}}$ & 3.8 & $79.9^{d}$ & 1.6 & $120.6^{c}$ & 3.5 \\
\hline \multicolumn{11}{|c|}{ Sports activity $\left(\mathrm{h}\right.$ week $\left.{ }^{-1}\right) \ddagger$} \\
\hline None & 4053 & 2674 & $88.9^{\mathrm{a}, \mathrm{c}}$ & 2.2 & $143.8^{\mathrm{a}}$ & & $91.3^{\mathrm{a}}$ & 2.1 & $139.0^{\mathrm{a}}$ & 4.2 \\
\hline$>0-2$ & 3827 & 2255 & $90.2^{a, c}$ & 2.3 & $140.8^{a}$ & 4.8 & $90.7^{a}$ & 2.2 & $135.3^{\mathrm{a}, \mathrm{c}}$ & 4.4 \\
\hline$>2-4$ & 4557 & 1742 & $86.3^{b, c}$ & 2.3 & $138.6^{a, b}$ & 5.0 & $86.3^{\mathrm{b}}$ & 2.2 & $134.1^{\mathrm{a}, \mathrm{c}}$ & 4.6 \\
\hline$>4-8$ & 3189 & 1873 & $84.8^{\mathrm{b}}$ & 2.4 & $138.2^{\mathrm{a}, \mathrm{b}}$ & 5.0 & $83.8^{\mathrm{b}}$ & 2.3 & $131.4^{\mathrm{b}, \mathrm{c}}$ & 4.6 \\
\hline$>8$ & 2969 & 2188 & $82.8^{\mathrm{b}}$ & 2.7 & $132.3^{\mathrm{b}}$ & 5.3 & $83.3^{\mathrm{b}}$ & 2.6 & $126.0^{\mathrm{c}}$ & 4.9 \\
\hline
\end{tabular}

*Adjusted for recall day, centre, age class, BMI class, smoking, education and sports activity, with or without adjustment for total energy intake (continuous). †Different superscripts indicate significantly different means within gender, factor and model. Vice versa, means with identical superscripts are not significantly different from each other within gender, factor and model; LSD-test, $P<0.05$.

$\ddagger$ Without data from Norway.

quality check at the end of the interview (in the presence of the subjects) ${ }^{22}$. In the present paper these data are used to calculate energy and fat intakes.

The present estimate deals with the intake of the food group 'meat and processed meat' (EPIC-SOFT food group 'meat'). Figure 1 provides an overview of food sub-groups contained entirely in EPIC-SOFT or newly reclassified in the case of processed meat; the building of summarised groups (sum of red meat, sum of poultry) is described as well. The EPIC-SOFT food sub-group 'fresh meat' includes all meat that has not been treated (preserved, cooked), but includes meat that has been minced or frozen (when bought). On the other hand, all meat that has experienced further treatment, such as preservation by salting and smoking, marinating or heating, or that has been bought as a ready-to-eat product (unknown recipe), was attributed to the EPIC-SOFT food sub-group 'processed meat'. This resulted in a very heterogeneous food sub-group. It was decided to separate ham, bacon and sausages from processed minced meat (such as hamburger, fricadel, meat balls) and processed meat cuts (e.g. 'schnitzel', slices of cold roasted meat, roasted meat in aspic). Usually, most minced meat and meat cuts were attached to fresh meat (mainly pork and beef, being part of a recipe); however, when bought as a ready-to-eat product, it was attributed to processed meat. Therefore, ham, bacon and sausages 


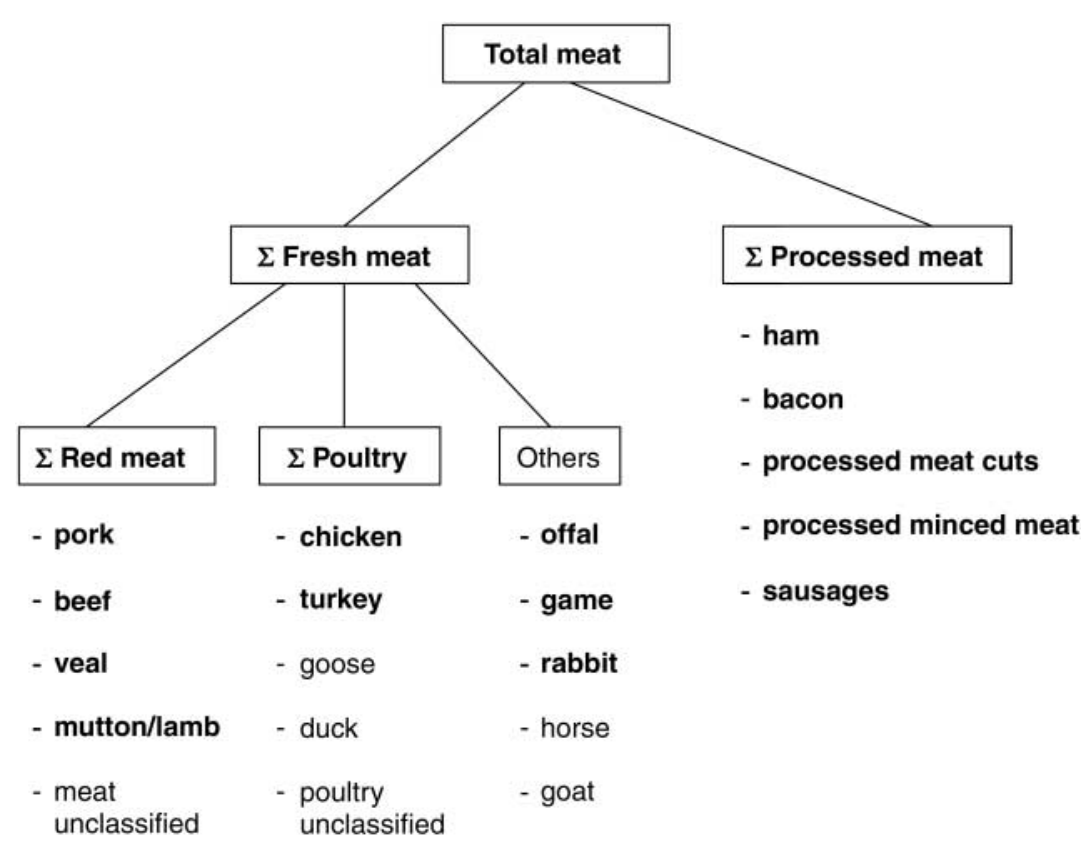

Fig. 1 Definition of sub-groups within the food group 'meat' (sub-groups in bold are given in Tables 1-3)

represent the original group of processed meat. For reclassification of processed meat, support from experts of each participating country was obtained. The information available on the food items was not sufficient to give a reliable estimate for the type of meat from which the processed meat was made; i.e. no categorisation such as 'processed meat prepared from red meat' was possible.

The summary variable 'red meat' includes pork, beef, veal and mutton/lamb. Veal was included in this term because the composition of veal in terms of its nutrient content is closer to that of beef than to other kinds of meat, although recognising that this varies according to differences in feeding practices and mean slaughtering age found in Europe. Moreover, in the Mediterranean countries veal is largely substituted for beef. The grouping applied here of veal as red meat should not be regarded as final but may depend on the questions addressed. The category 'red meat' also contains all unclassified fresh meat items because these refer mainly to mixed (pork and beef) minced meat.

Crude intake values are given as arithmetic means; adjusted values are presented as mean and standard error of the mean. All conclusions given are based on the adjusted values. Adjustment within centres or countries was performed to correct for deviations from an ideal sampling of the 24-hour recalls (day of the week, season) as well as for age. For days of the week, two discrete levels (Monday-Friday, Saturday-Sunday) and for season four discrete levels were applied (weighting). Age was included as a continuous variable. Furthermore, the presentation of the results is stratified for sex and centre. For graphical presentations, most centres were aggregated into regions or countries whenever possible. Due to the distinct differences in mean intake data between centres in
Spain and Italy, two regions were derived for Spain (south and north) and only one for Italy (north), keeping the two southern Italian centres separate. In order to consider the differences in total energy intake, the percentage of total daily energy intake provided by consumption of meat was calculated. Similarly, the contribution of meat intake to the total daily fat intake was estimated.

Factors significantly affecting total meat consumption were identified by means of analysis of variance. Testing of statistical significance between groups was performed with the least square differences (LSD) test at an $\alpha$ level of $5 \%$. The factors centre, day of the week and season of the recall assessment, sex, age, body mass index (BMI), smoking, education, physical activity at work and sports activity were tested. Information on the latter four variables was collected by means of questionnaires and interviews at recruitment and detailed information on these parameters is given elesewhere ${ }^{19,23}$. Calculation of adjusted values was performed using SAS System ${ }^{\circledR}$ for Windows $^{\mathrm{TM}}$ Release 8.00 (SAS Institute Inc., Cary, NC, USA). All other calculations were done by means of SPSS ${ }^{\circledR}$ for Windows ${ }^{\mathrm{TM}}$ Release 10.0.7 (SPSS Inc., Chicago, IL, USA).

\section{Results}

\section{Total meat}

Quite a high variation in meat consumption across EPIC was observed (Tables $1 \mathrm{a}$ and 1b, Figs $2 \mathrm{~A}$ and $2 \mathrm{~B}$ ). Except for the 'health-conscious' cohort in the UK, adjusted mean total meat intake was lowest in Greece ( 47 and $79 \mathrm{~g} \mathrm{day}^{-1}$ in women and men, respectively) and highest in the north of Spain, especially in San Sebastian (124 and $234 \mathrm{~g} \mathrm{day}^{-1}$, respectively). Besides San Sebastian, the highest mean 
Meat consumption in EPIC
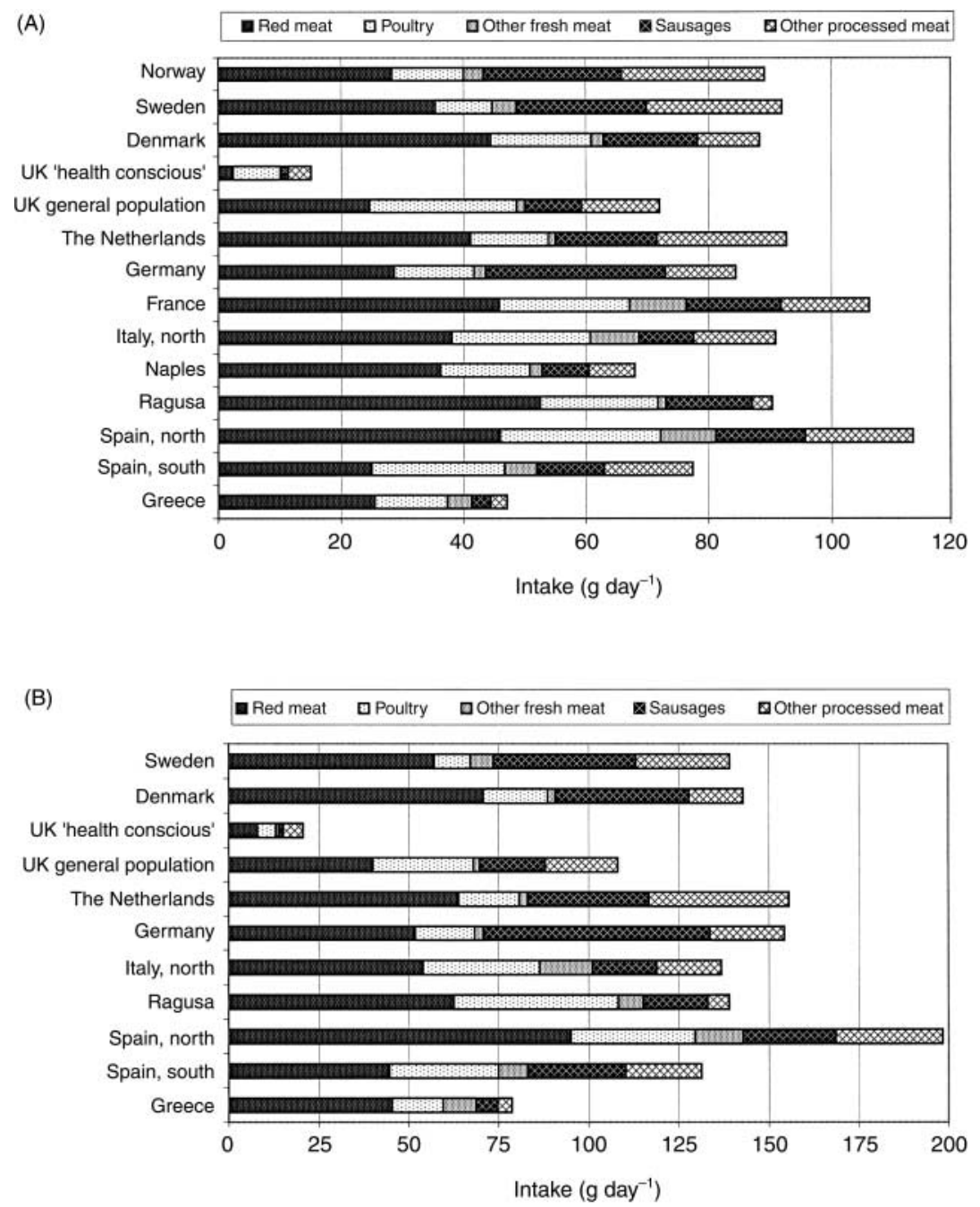

Fig. 2 Mean intakes of total meat and meat sub-groups ( $\mathrm{g} \mathrm{day}^{-1}$, adjusted $\left.{ }^{\star}\right)$ in women $(\mathrm{A})$ and men (B) across 10 European countries participating in the European Investigation into Cancer and Nutrition (EPIC) calibration study (24-hour recalls). *Adjusted for age as well as for day of the week and season of the 24-hour recall assessment

total meat intake among the EPIC centres was in the other northern Spanish centres (Asturias, Navarra). This contrasts with the situation found in the south of Spain (Granada, Murcia), with a distinctly lower consumption of red meat, especially veal and beef. On average, the total meat intake in Naples (Italy) was lower than in the more northern EPIC centres from Italy as a consequence of a lower intake of fresh meat (beef, poultry) as well as processed meat. However, Ragusa (Sicily) does not fit with this south-north distinction, with meat intake values more comparable to those in the northern Italian centres. Among the southern European EPIC centres, participants from Greece revealed the lowest total meat intake figures.

The meat intake pattern in the 'health-conscious' population of the UK, including about $70 \%$ who ate little or no meat (vegans, ovo-lacto vegetarians, fish eaters), is greatly different from that of all other EPIC centres. While this group ate nearly no meat or processed meat, the remaining 30\% in this very special British cohort reported a distinctly lower mean meat intake (women, $44 \mathrm{~g} \mathrm{day}^{-1}$; men, $79 \mathrm{~g} \mathrm{day}^{-1}$ ) than the general population in the UK. Other than for the EPIC centres in Spain, Italy and the UK, no major differences in total meat intake between EPIC centres within the other countries (France, The Netherlands, Germany, Denmark, Sweden and Norway) were observed. Except for the 'health-conscious' EPIC cohort in the UK, the general population of the UK showed the lowest mean total meat intake among the middle and northern European EPIC centres, while meat intake patterns (quantity and quality, i.e. the proportions of meat sub-groups) were to some extent comparable between The Netherlands, Germany, Denmark, Sweden and Norway.

\section{Red meat}

Red meat intake varied from 24 to $57 \mathrm{~g} \mathrm{day}^{-1}$ in women and from 40 to $121 \mathrm{~g} \mathrm{day}^{-1}$ in men (Table 1). The corresponding intake figures for the 'health-conscious' cohort in the UK were $3 \mathrm{~g} \mathrm{day}^{-1}$ in women and $8 \mathrm{~g} \mathrm{day}^{-1}$ in men. 
Most pork was consumed in the German (24 and $36 \mathrm{~g} \mathrm{day}^{-1}$ in Potsdam for women and men, respectively), Swedish, Danish and Dutch EPIC centres. Intake of beef/veal and mutton was highest in the EPIC centres of northern Spain (39 and $104 \mathrm{~g} \mathrm{day}^{-1}$ for women and men, respectively, in San Sebastian), the Italian centres and the French centres. Poultry (mostly chicken) consumption tended to be higher in the southern European centres (except Greece and Naples, Italy) and the general population cohort of the UK, compared with the more northern EPIC centres of Europe.

\section{Rarely consumed meat}

For meat rarely consumed, i.e. game, rabbit, horse, goat, duck and goose, intake data were calculated at the country level. Rabbit consumption was highest in the EPIC cohorts of France (3.2 $\mathrm{g} \mathrm{day}^{-1}$, women only), Italy (2.8 and $5.2 \mathrm{~g} \mathrm{day}^{-1}$ in women and men, respectively) and Spain (2.5 and $3.9 \mathrm{~g} \mathrm{day}^{-1}$, respectively), while game consumption was highest in the EPIC centres of Sweden (3.9 and $5.6 \mathrm{~g} \mathrm{day}^{-1}$, respectively) and Norway (2.3 $\mathrm{g} \mathrm{day}^{-1}$, women only). A country-specific preference was observed for intake of goat in the Greek EPIC cohort (1.5 and $2.8 \mathrm{~g} \mathrm{day}^{-1}$ for women and men, respectively).

\section{Processed meat}

Tables $2 \mathrm{a}$ and $2 \mathrm{~b}$ show the results for processed meat intake. The EPIC-SOFT food group 'processed meat' was reclassified in order to derive valid data on the intake of ham, bacon and sausages. For sausages, the German EPIC centres revealed the highest mean intake in both women and men (41 and $87 \mathrm{gday}^{-1}$ in Potsdam, respectively), followed by the cohorts in Norway (women only), Sweden, Denmark and The Netherlands. In EPIC Greece, Italy (except Ragusa) and the UK, average sausage consumption was distinctly lower. Also ham consumption was lowest in the Greek EPIC cohort and increased in the order of Ragusa (Italy), Potsdam (Germany), Denmark and the UK (general population). While intake figures for bacon were quite low in most EPIC centres, bacon amounted to about $25 \%$ of processed meat intake in the general population of EPIC UK.

\section{Removal of visible fat}

During the interview, participants could indicate whether or not the meat item was consumed with or without visible fat. In about $20 \%$ of all consumption occurrences, EPIC participants from France (women only), Italy, Spain and the UK indicated the removal of visible fat from fresh meat before consumption; indications in other EPIC countries varied between $8 \%$ and $15 \%$. For processed meat, most indications of fat removal occurred in the French (29\% of all processed meat consumption occurrences) and British (23\%, general population) EPIC cohorts, followed by Spanish (16\%) and Norwegian (13\%); in all other EPIC countries, indications were below $8 \%$. Of course, the possibility to remove fat is restricted to food items with visible fat; i.e. in the group of processed meat, mainly ham and other processed meat cuts. The clear effect of this practice should be visible at the nutrient level (i.e. lipid intake) only, because the figures presented do not consider the total amount of an item consumed but only whether or not it is consumed. Therefore, in Tables $3 \mathrm{a}$ and $3 \mathrm{~b}$, data on the contributions of meat and meat sub-groups to the total lipid intake are provided.

\section{Modifiers of meat intake}

Factors significantly affecting meat consumption were sex, age, BMI, centre, day of dietary recall, vegetarian status, smoking behaviour, education, and total energy intake. Meat consumption was most strongly associated with total energy intake; therefore, intakes of total meat and the main meat sub-groups were calculated in terms of the percentage of total energy intake (Table 4, Figs $3 \mathrm{~A}$ and 3B). As compared with the intake data in grams per day, the differences between centres changed. For example, women from Naples became comparable in total meat intake to the other Italian EPIC centres, Granada is no longer the Spanish EPIC centre with the lowest meat intake, but in the range of San Sebastian, while Asturias moved to the low end of Spanish EPIC centres. Although the Greek cohort is still at the second lowest place in meat intake, the difference from other EPIC centres, e.g. the general population cohort in the UK, became much smaller than found for the intake data in grams per day.

Over the whole study population, 276 women and 136 men stated they were vegetarians, most of them originating from the British cohort of 'health-conscious' people (130 women and 89 men). The vegetarians within the 'health-conscious' British cohort consumed on average only $1.4 \mathrm{~g} \mathrm{day}^{-1}$ of meat and meat products. Regarding other centres, the highest number of vegetarians was included in the German EPIC cohorts with 45 women and 23 men. The range of mean total meat intake in vegetarians of other countries varied from 0 to $27.6 \mathrm{~g} \mathrm{day}^{-1}$ (Greece, $n=4$ ). Because of the low number of vegetarians in this study, the results could not be adjusted for vegetarian status.

Sunday was the day with the highest meat consumption; on the other hand, meat intake was lowest on Friday (Table 4). With increasing age the amount of meat consumed decreased significantly, in both women and men. Participants with a higher relative body weight (BMI) ate more meat and meat products than subjects with a low BMI. Also, smoking habit showed an effect on meat intake, with smokers revealing a significantly higher intake of meat than former or never smokers. With increasing educational level and sports activity, the meat intake values decreased. Adjustment for total energy intake did not change these results in general (Table 4). 


\section{Discussion}

This is one of the first reports comparing quantitative intake estimates of meat and meat products across Europe, as assessed by means of a highly standardised dietary assessment tool. The mean results of the 24-hour dietary recalls should represent reliable estimates at the group level for comparisons between European centres participating in EPIC. The EPIC cohorts are not representative samples of the population, either of the centre or the host country (with few exceptions, e.g. Norway). However, the population is sufficiently large to serve as a basis for reference data. The first purpose of the calibration study was to obtain a good estimate of food intakes at the population level, and only a single 24-hour dietary recall measurement was collected from each study subject who participated. The analysis is therefore focused on the comparison of mean dietary estimates rather than medians, although recognising that the distribution of intakes of individuals is mostly skewed. All descriptive papers on food intake, as assessed by the 24-hour dietary recalls in EPIC, were adjusted for unbalanced sampling of the 24-hour recalls (distribution over days of the week and seasons) and for age. Analysis of variance confirmed a significant effect of age and day of the week on total meat consumption (Table 4).

As expected, energy intake was found to be strongly associated with meat consumption. The 24-hour dietary recalls were checked at the end of the interview for extreme energy intake values and corrected, if necessary, in the presence of the subject. However, no control instrument was available, particularly for underreporting $^{22}$. The extent of bias introduced by neglecting to mention foods or underestimating portion sizes is discussed in detail elsewhere in this supplement ${ }^{24}$. However, in terms of meat intake, energy adjustment resulted in considerably higher values for the EPIC participants in Greece. This suggests that underreporting
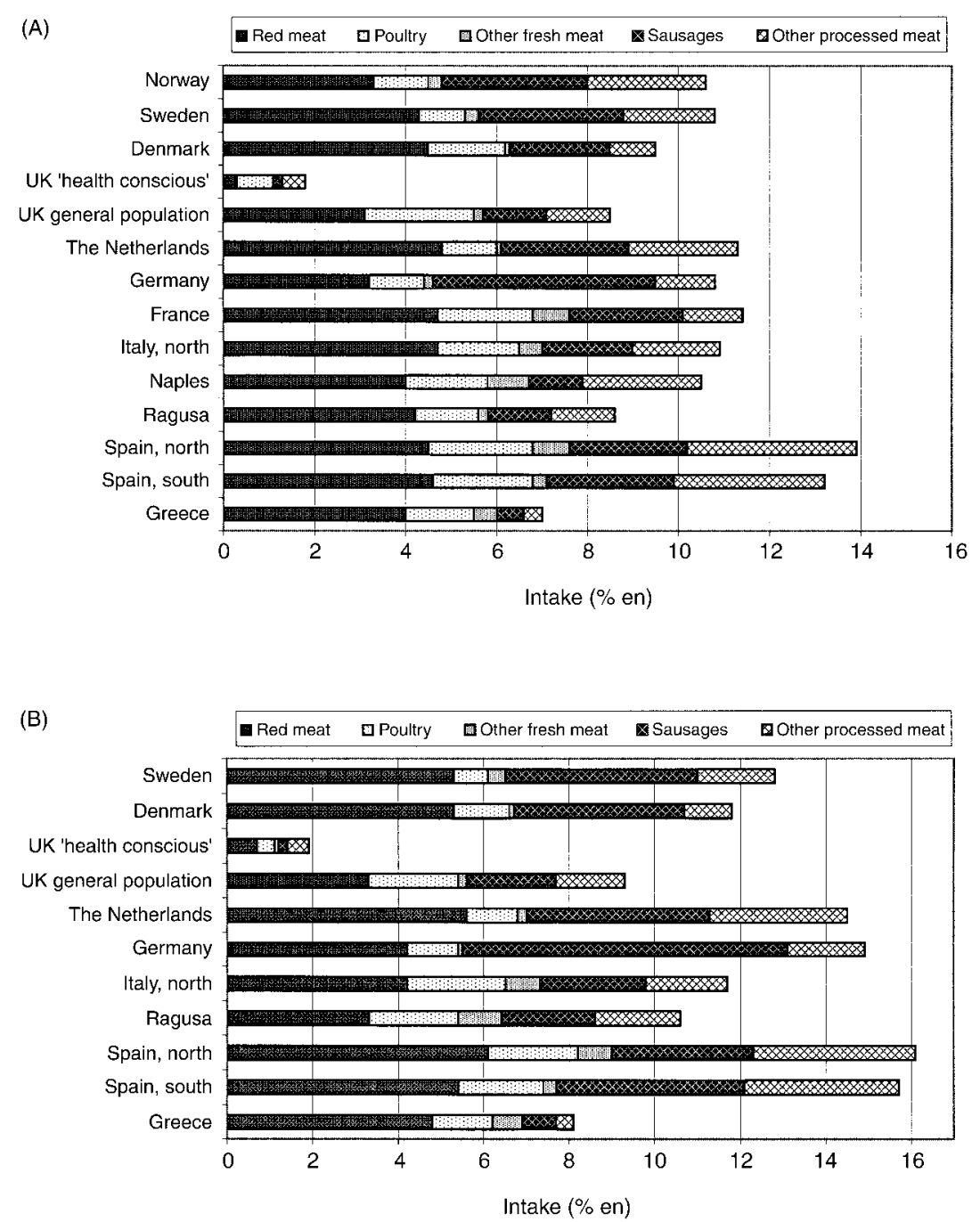

Fig. 3 Mean contributions of the food group 'meat' and its sub-groups to total energy intake (\% en, adjusted $)$ in women (A) and men (B) across 10 European countries participating in the European Investigation into Cancer and Nutrition (EPIC) calibration study (24-hour recalls). ${ }^{*}$ Adjusted for age as well as for day of the week and season of the 24-hour recall assessment) 
in the Greek cohort is more extensive than in other EPIC centres, in both men and women. Comparing the meat intake data (not adjusted for energy) with the household budget survey data from Greece (DafneSoft v1.0, Department of Epidemiology, University of Athens Medical School, Athens, Greece), a discrepancy is evident. While Greek total meat availability in 1994 was comparable to or in some cases higher than the availability in most other European countries, the present study revealed intakes below those found in other EPIC centres. Next to EPIC-Greece, a similar situation was found for the southern Spain centres. The distinct differences in meat intake between the southern and northern Spanish centres diminished after energy adjustment (Figs 2 and 3); these differences are likely to be the result of a higher proportion or extent of underreporting in the southern Spanish centres.

The impact of the bovine spongiform encephalopathy (BSE) crisis on quantitative and qualitative meat consumption patterns is rarely described ${ }^{25}$. No measure of changes in meat consumption habits through awareness of BSE was included in the 24-hour recalls. Since the first cases of BSE were identified in British cows in the late 1980s, the BSE problem has remained unsolved until now $^{26}$. The effect on consumer attitudes towards beef consumption (or meat consumption in general) may vary by country and time point. However, the public awareness of the BSE crisis reached a peak in early 1996 when the European Commission prohibited the export of British bovine animals and their products. During the first half of 1996, the EPIC calibration study was already started or ongoing in France, Italy, Spain, The Netherlands and Malmö (Sweden). In all other EPIC centres, data collection started in the second half of 1996 or later ${ }^{18}$.

The relatively low meat intake data obtained for the general population in EPIC-UK did not change substantially by energy adjustment. According to data from the World Health Organization/Food and Agriculture Organization, meat (red meat) consumption in the UK has decreased in the past three decades by about $25 \%$ to values lower than those found in the Mediterranean regions ${ }^{27}$. A recent description of dietary behaviour in Central England and a French Mediterranean region demonstrated that meat and processed meat were less frequently consumed in England than the South of France $^{28}$. For example, $28 \%$ of the French sample and $1.9 \%$ of the UK samples reported eating red meat at least once a day. This fits with the results obtained in these EPIC cohorts. As far as recent national intake data on meat and meat sub-groups are available, comparisons with the data presented here showed good agreement ${ }^{29-36}$.

Denmark has been described as the country with the highest intake of meat and red meat in Europe or even in the world $^{6}$. However, a decrease in meat consumption has been reported ${ }^{30,33}$ and is confirmed by these data: total meat intake in the Danish EPIC centres was comparable to those obtained for the EPIC cohorts in other Nordic countries as well as in Germany, The Netherlands, northern Italy and Ragusa. Regarding red meat, the Danish centres were among those with the highest intakes, along with the EPIC centres in the north of Spain, France and Ragusa. A low intake of red meat together with a preference for veal is described as a characteristic of the traditional Mediterranean $\operatorname{diet}^{37-39}$. Accordingly, in the present study the lowest intake of red meat was reported by EPIC participants in the south of Spain, Naples and Greece, but not in the South of France or Ragusa. Veal is a prominent source of meat in Greece, Spain, Italy and France only.

Distinct differences between EPIC centres were observed for processed meat. Due to its heterogeneity, this food sub-group was subdivided into sausages, ham and bacon as well as other processed meat products (minced meat, meat cuts). Germany had the highest intake of sausages by far, followed by the Nordic countries and The Netherlands. A more detailed characterisation of processed meat according to preservation and cooking techniques as well as the provision of nutrients will be published elsewhere (in preparation).

The literature provides several reports on differences in meat consumption by gender and socio-economic status $^{2,40,41}$. For example, in Norway, a diminishing social value of meat and hence a trend towards decreasing meat intake exists particularly among well-educated women, contrasting with the fact that meat consumption may still be considered as a status symbol in lower socio-economic groups ${ }^{41}$. Owing to the greater activity of women in gaining health-related information and implementing this in daily life, public dietary recommendations may be observed earlier and more strikingly in women than in men. In EPIC, gender differences in meat intake were quite striking and can be explained only partly by different energy intakes in men and women. The data also demonstrate that meat intake decreases as educational level (as a measure of socio-economic status) increases (Table 4; except for women in the lowest education group). Because of the low number of self-stated vegetarians overall (except in the 'health-conscious' group recruited in the UK), a higher prevalence of vegetarianism and meat avoidance in women and in higher social classes ${ }^{42}$ was not evident.

Far before the BSE crisis, the popularity of meat and meat products in several Western countries - including the UK, Norway and Denmark - was in a process of decline $^{2}$. Negative attitudes towards meat are frequently expressed, which, however, are not necessarily associated with decreased meat consumption. One of the most often cited critical attitudes pertains to the perceived unhealthiness of meat consumption. A major recommendation for reducing the risk of cardiovascular disease is to limit intake of total fat, especially saturated fat, and cholesterol. Since meat and meat products may contribute substantially to the supply of these nutrients, the dietary advice is to 
choose lean meat over fat ${ }^{4}$. Moreover, haem iron has been considered for possible involvement in disease aetiology via its oxidative potential ${ }^{11}$. On the other hand, dietary recommendations for cancer prevention may include distinct figures for restriction of red meat intake to less than 80 g daily $^{6}$, although this limit is not generally agreed ${ }^{27}$. The rationale for such a recommendation in limiting meat intake refers to the fat and iron contents of meat and processed meat. Furthermore, human exposure to carcinogens or suspected carcinogens produced during cooking, such as heterocyclic amines, polycyclic aromatic amines and $\mathrm{N}$-nitroso compounds, is modulated by meat intake. Presently, most epidemiological evidence is available for a detrimental role of meat and meat products in the aetiology of colorectal cancer. A role of meat intake in cancer development is also discussed for cancers of the breast, pancreas, prostate and kidney ${ }^{6}$. Detailed information on how far dietary recommendations for cancer prevention were met by the participants of the entire EPIC calibration study will be provided in a forthcoming publication.

The fat content of meat ranges from a low of less than $4 \%$ for lean poultry to $30-40 \%$ fat by weight for fatty meat from domesticated farmed animals; wild meat is lower in fat. About half of the fatty acids in meat comprise saturated fatty acids (SFA; except poultry with about 35\% SFA). For several European countries, data on the contribution of meat to the total daily energy and fat intakes have been reported $^{43-45}$. Although direct comparison is difficult due to differences in food group aggregation, considerable deviations across European countries can be expected. This is confirmed by the EPIC data on the contribution of meat intake to total energy and fat intakes (Table 3). For example, $0.8-8.0 \%$ of total energy intake and $0.8-15.6 \%$ of total fat intake is provided by sausage consumption in EPIC men (without consideration of the 'health-conscious' EPIC cohort in the UK). However, nutrient data (energy, fat) have to be interpreted cautiously since they may differ by the food composition table used ${ }^{46}$. Full comparability of nutrient data across centres would only be better by means of a common European food composition table ${ }^{47}$.

In conclusion, the high variation in meat intake observed in EPIC centres across Europe reflects the development of consumer habits and attitudes during the past decades. Even higher differences exist at the meat sub-group level, e.g. for red meat or processed meat intake. This provides a good opportunity for EPIC to strengthen our knowledge on the role of meat in the aetiology of chronic diseases. In light of the BSE crisis, recording changes in meat consumption habits might be necessary in order to prevent bias in long-term risk evaluation.

\section{Acknowledgements}

The work described in this paper was carried out with financial support of the 'Europe Against Cancer'
Programme of the European Commission (SANCO); Ligue contre le Cancer (France); Société 3M (France); Mutuelle Générale de l'Education Nationale; Institut National de la Santé et de la Recherche Médicale (INSERM); Institute Gustave Roussy; German Cancer Aid; German Cancer Research Centre; German Federal Ministry of Education and Research; Danish Cancer Society; Health Research Fund (FIS) of the Spanish Ministry of Health; the Spanish Regional Governments of Andalucia, Asturias, Basque Country, Murcia and Navarra; Cancer Research UK; Medical Research Council, UK; Stroke Association, UK; British Heart Foundation; Department of Health, UK; Food Standards Agency, UK; Wellcome Trust, UK; Greek Ministry of Health; Greek Ministry of Education; Italian Association for Research on Cancer; Italian National Research Council; Dutch Ministry of Public Health, Welfare and Sports; Dutch Prevention Funds; LK Research Funds; Dutch ZON (Zorg Onderzoek Nederland); World Cancer Research Fund; Swedish Cancer Society; Swedish Scientific Council; Regional Government of Skane, Sweden; Norwegian Cancer Society; Norwegian Research Council. Partial support for the publication of this supplement was provided by the Centre de Recherche et d'Information Nutritionnelles (CERIN).

In addition, we wish to thank all study participants for their co-operation and all interviewers who participated in the fieldwork studies in each EPIC centre.

\section{References}

1 Mann N. Dietary lean red meat and human evolution. Eur. J. Nutr. 2000; 39: 71-9.

2 Holm L, Mohl M. The role of meat in everyday food culture: an analysis of an interview study in Copenhagen. Appetite 2000; 34: 277-83.

3 Sugimura T. Nutrition and dietary carcinogens. Carcinogenesis 2000; 21: 387-95.

4 Kraus RM, Eckel RH, Howard B, Appel LJ, Daniels SR, Deckelbaum RJ, et al. AHA dietary guidelines. Revision 2000: a statement for healthcare professionals from the Nutrition Committee of the American Heart Association. Circulation 2000; 102: 2284-99.

5 Bingham SA. Diet and colorectal cancer prevention. Biochem. Soc. Trans. 2000; 28: 12-6.

6 World Cancer Research Fund/American Institute for Cancer Research (WCRF/AICR). Food, Nutrition and the Prevention of Cancer: A Global Perspective. Washington, DC: WCRF/AICR, 1997.

7 Committee on Medical Aspects of Food and Nutrition Policy (COMA). Nutritional Aspects of the Development of Cancer. Report of the Working Group on Diet and Cancer. Report on Health and Social Subjects No. 48. Norwich, UK: The Stationary Office, 1998.

8 Norat T, Riboli E. Meat consumption and colorectal cancer: a review of epidemiologic evidence. Nutr. Rev. 2001; 59: $37-47$.

9 Sandhu MS, White IR, McPherson K. Systematic review of the prospective cohort studies on meat consumption and colorectal cancer risk: a meta-analytical approach. Cancer Epidemiol. Biomark. Prev. 2001; 10: 439-46.

10 Truswell AS. Report of an expert workshop on meat intake and colorectal cancer risk convened in December 1998 in 
Adelaide, South Australia. Eur. J. Cancer Prev. 1999; 8 $175-8$.

11 Sesink AL, Termont DS, Kleibeuker JH, Van Der Meer R. Red meat and colon cancer: dietary haem, but not fat, has cytotoxic and hyperproliferative effects on rat colonic epithelium. Carcinogenesis 2000; 21: 1909-15.

12 Bingham SA. High-meat diets and cancer risk. Proc. Nutr Soc. 1999; 58: 243-8

13 Danesh J, Appleby P. Coronary heart disease and iron status. Meta-analyses of prospective studies. Circulation 1999; 99 $852-4$.

14 Grant WB. The role of meat in the expression of rheumatoid arthritis. Br. J. Nutr. 2000; 84: 589-95.

15 Trichopoulou A, Lagiou P. The DAFNE food data bank as a tool for monitoring food availability in Europe. Data Food Networking. Public Health Rev. 1998; 26: 65-71.

16 World Health Organization (WHO). Diet, Nutrition and the Prevention of Chronic Diseases. Report of the WHO Study Group. Technical Report Series No. 797. Geneva: WHO, 1990.

17 Schroll K, Moreiras-Varela O, Schlettwein-Gsell D, Decarli B, de Groot L, van Staveren W. Cross-cultural variations and changes in food-group intake among elderly women in Europe: results from the Survey in Europe on Nutrition and the Elderly: a Concerted Action (SENECA). Am.J. Clin. Nutr. 1997; 65(Suppl. 4): 1282S-9S.

18 Riboli E, Hunt KJ, Slimani N, Ferrari P, Norat T, Fahey R, et al. European Prospective Investigation into Cancer and Nutrition (EPIC): study populations and data collection. Public Health Nutr. 2002; 5(6B): 1113-24.

19 Slimani N, Kaaks R, Ferrari P, Casagrande C, Clavel-Chapelon F, Lotze G, et al. European Prospective Investigation into Cancer and Nutrition (EPIC) calibration study: rationale, design and population characteristics. Public Health Nutr. 2002; 5(6B): $1125-45$.

20 Slimani N, Deharveng G, Charrondière RU, van Kappel AL, Ocke MC, Welch A, et al. Structure of the standardized computerized 24-h diet recall interview used as reference method in the 22 centres participating in the EPIC project. European Prospective Investigation into Cancer and Nutrition. Comput. Meth. Programs Biomed. 1999; 58: 251-66.

21 Slimani N, Ferrari P, Ocké M, Welch A, Boeing H, van Liere $\mathrm{M}$, et al. Standardization of the 24-hour diet recall calibration method used in the European Prospective Investigation into Cancer and Nutrition (EPIC): general concepts and preliminary results. Eur. J. Clin. Nutr. 2000; 54: 900-17.

22 Voss S, Charrondière UR, Slimani N, Kroke A, Riboli E, Wahrendorf $\mathrm{J}$, et al. EPIC-SOFT: a European computer program for 24-hour dietary protocols. Z. Ernahrungswiss. 1998; 37: 227-33.

23 Haftenberger M, Schuit AJ, Tormo MJ, Boeing H, Wareham $\mathrm{N}$, Bueno-de-Mesquita HB, et al. Physical activity of subjects aged 50-64 years involved in the European Prospective Investigation into Cancer and Nutrition (EPIC). Public Health Nutr. 2002; 5(6B): 1163-77.

24 Ferrari P, Slimani N, Ciampi A, Trichopoulos D, Naska A, Lauria C, et al. Evaluation of under- and overreporting of energy intake in the 24-hour diet recalls in the European Prospective Investigation into Cancer and Nutrition (EPIC). Public Health Nutr. 2002; 5(6B): 1329-45.

25 Morabia A, Bernstein MS, Heritier S, Beer-Borst S. A Swiss population-based assessment of dietary habits before and after the March 1996 'mad cow' crisis. Eur. J. Clin. Nutr. 1999; 53: $158-63$

26 Aldhous P. Inquiry blames missed warning for scale of Britain's BSE crisis. Nature 2000; 408: 3-5.

27 Hill M. Meat and colorectal cancer: a European perspective. Eur. J. Cancer Prev. 1999; 8: 183-4.
28 Holdsworth M, Gerber M, Haslam C, Scali J, Beardsworth A, Avallone $\mathrm{MH}$, et al. A comparison of dietary behaviour in Central England and a French Mediterranean region. Eur. J. Clin. Nutr. 2000; 54: 530-9.

29 Haraldsdottir J. Dietary guidelines and patterns of intake in Denmark. Br. J. Nutr. 1999; 81(Suppl. 2): S43-8.

30 Groth MV, Fagt S. Trends in dietary habits in Denmark and Sweden since the 1960s. Ugeskr. Laeger. 2001; 163: 425-9.

31 Adolf T, Scheider R, Eberhardt W, Hartmann S, Herwig A, Heseker $\mathrm{H}$, et al. Ergebnisse der Nationalen Verzehrsstudie (1995-1988) über die Lebensmittel- und Nährstoffaufnabme in der Bundesrepublik Deutschland. VERA-Schriftenreihe XI. Niederkleen, Germany: Wissenschaftlicher Fachverlag Dr. Fleck, 1995.

32 Elmstahl S, Holmqvist O, Gullberg B, Johansson U, Berglund G. Dietary patterns in high and low consumers of meat in a Swedish cohort. Appetite 1999; 32: 191-206.

33 Osler M, Heitmann BL, Schroll M. Ten-year trends in the dietary habits of Danish men and women. Cohort and crosssectional data. Eur. J. Clin. Nutr. 1997; 51: 535-41.

34 Mensink GBM, Thamm M, Haas K. Die Ernährung in Deutschland 1998. Gesundheitswesen 1999; 61(Suppl. 2): S200-6.

35 Alberti-Fidanza A, Fidanza F, Chiuchiu MP, Verducci G, Fruttini D. Dietary studies on two rural Italian population groups of the Seven Countries Study. 3. Trend of food and nutrient intake from 1960 to 1991. Eur.J. Clin. Nutr. 1999; 53 854-60.

36 Anonymous. Zo eet Nederland. Resultaten van den Voedselconseumptie-peiling 1997-1998. Den Haag, The Netherlands: Voedingscentrum, 1998; 15.

37 Trichopoulou A, Lagiou P, Kuper H, Trichopoulos D. Cancer and Mediterranean dietary traditions. Cancer Epidemiol. Biomark. Prev. 2000; 9: 869-73.

38 Trichopoulou A, Vasilopoulou E. Mediterranean diet and longevity. Br. J. Nutr. 2000; 84(Suppl. 2): S205-9.

39 Kushi LH, Lenart EB, Willett WC. Health implications of Mediterranean diets in light of contemporary knowledge. 2. Meat, wine, fats, and oils. Am.J. Clin. Nutr. 1995; 61(Suppl.): 1416S-27S.

40 Lea E, Worsley A. Influences on meat consumption in Australia. Appetite 2001; 36: 127-36.

41 Fagerli RA, Wandel M. Gender differences in opinions and practices with regard to a 'healthy diet'. Appetite 1999; 32 : $171-90$.

42 Santos MLS, Booth DA. Influences on meat avoidance among British students. Appetite 1996; 27: 197-205.

43 Winkler G, Döring A, Keil U. Trends in dietary sources of nutrients among middle-aged men in southern Germany. Results of the MONICA Project Augsburg: dietary surveys 1984/1985 and 1994/1995. Appetite 2000; 34: 37-45.

44 Cade JE, Margetts BM. Nutrient sources in the English diet: quantitative data from three English towns. Int. J. Epidemiol. 1988; 17: 844-8.

45 Freudenheim JL, Krogh V, D'Amicis A, Scaccini C, Sette S, Ferro-Luzzi A, et al. Food sources of nutrients in the diet of elderly Italians: I. Macronutrients and lipids. Int.J. Epidemiol. 1993; 22: 855-68.

46 Deharveng G, Charrondière UR, Slimani N, Southgate DA, Riboli E. Comparison of nutrients in the food composition tables available in the nine European countries participating in EPIC. Eur. J. Clin. Nutr. 1999; 53: 60-79.

47 Slimani N, Charrondière UR, van Staveren W, Riboli E. Standardization of food composition databases for the European Investigation into Cancer and Nutrition (EPIC): general theoretical concept. J. Food Comp. Anal. 2000; 13 $567-84$. 